\title{
Angio-Associated Migratory Cell Protein (AAMP) Regulates the Hippo/YAP Pathway and Mitochondrial Functionality to Drive Osteosarcoma Metastasis
}

\section{Fangbiao Zhan}

Chongqing University Three Gorges Hospital

\section{Zhiyu Chen}

The First Affiliated Hospital of Chongqing Medical University

\section{Chaozheng Xie}

The First Affiliated Hospital of Chongqing Medical University

\section{Shuang Xiang}

Chongqing University Three Gorges Hospital

\section{Qianrong Deng}

Chongqing University Three Gorges Hospital

\section{Huanhuan Li}

Chongqing University Three Gorges Hospital

\section{Lin Tian}

Chongqing University Three Gorges Hospital

\section{Changchun Jian}

The First Affiliated Hospital of Chongqing Medical University

\section{Jiangxia Xiang}

The First Affiliated Hospital of Chongqing Medical University

\section{Yunsheng Ou}

The First Affiliated Hospital of Chongqing Medical University

Jian Chen

Chongqing University Three Gorges Hospital

Lixin Xu (D WANGJIAPOTHREE456@163.com)

Chongqing University Three Gorges Hospital https://orcid.org/0000-0002-0282-1772

\section{Research article}

Keywords: Osteosarcoma, AAMP, YAP, Mitochondrial function, Metastasis, CFL1

Posted Date: January 3rd, 2022 
DOI: https://doi.org/10.21203/rs.3.rs-1208015/v1

License: (c) (1) This work is licensed under a Creative Commons Attribution 4.0 International License. Read Full License 


\section{Abstract}

Background: Osteosarcoma (OS) is the prevalent form of primary bone cancer among adolescents, but the 5 -year overall survival rate for patients with a metastatic or recurrent OS is under $20 \%$. Angioassociated migratory cell protein (AAMP) is known to be a key regulator of cellular migration, yet its role in the context of OS metastasis has yet to be firmly established.

Methods: Bioinformatics analyses were used to explore the association between AAMP and YAP expression and the prognosis of OS patients, and to evaluate differences in AAMP expression in patients with primary OS, recurrent OS, and pulmonary metastatic OS. Immunohistochemical (IHC) staining was additionally performed to compare AAMP levels in primary OS and pulmonary metastatic OS patient samples. Lentiviral transduction was further used to establish OS cell lines in which AAMP or YAP had been stably knocked down or overexpressed. OS cell migration and invasion were assessed using wound healing and Transwell assays. Proteins associated with the mitochondria, the epithelial-mesenchymal transition (EMT), YAP, and its target proteins were assessed in OS cell lines via Western blotting. OS cell lamellipodia were detected via phalloidin staining. Mitochondrial morphological characteristics were assessed via transmission electron microscopy following the knockdown of AAMP. An ATP kit was employed to measure ATP levels in OS cells in which AAMP had been knocked down. Animal model studies were used to confirm indices associated with OS cell lung metastasis following AAMP knockdown.

Results: Patients with metastatic OS exhibit higher levels of AAMP expression that are correlated with poorer patient prognosis. Knocking down AAMP suppressed the migratory, invasive, and EMT activity of analyzed OS cell lines. AAMP was found to regulate CFL1 and thereby control OS cell protrusion. AAMP knockdown was further found to promote OS cell mitochondrial dysfunction and decreased intracellular ATP production, with these AAMP knockdown cells exhibiting impaired migratory and invasive activity as a consequence of YAP inhibition. Consistently, the knockdown of AAMP suppressed the in vivo metastasis of OS cells.

Conclusions: Together, these data highlight a model wherein AAMP can promote OS cell migratory and invasive activity by regulating YAP and mitochondrial functionality. The AAMP/CFL1/YAP signaling pathway may thus represent a viable therapeutic target for efforts aimed at suppressing the metastatic progression of OS.

\section{Background}

Osteosarcoma (OS) is the most common form of primary bone malignancy among adolescents (Belayneh et al., 2021; Smrke et al., 2021). Despite extensive research efforts, therapeutic options for OS patients have largely remained unchanged over the last three decades and primarily consist of a combination of surgery and neoadjuvant chemotherapy (Smrke et al., 2021). In contrast to other solid tumor types, no targeted drugs have been approved for the treatment of OS (Meltzer and Helman, 2021). 
While patients with the localized disease exhibit a 5-year survival rate of roughly $60 \%$, for those with recurrent disease or pulmonary metastases this rate drops to 18.9\%-20\% (Bacci et al., 2008; Meltzer and Helman, 2021). It is thus critical that the mechanisms governing OS progression and metastasis be better clarified in order to identify novel biomarkers and therapeutic targets that can be leveraged to guide patient treatment efforts.

Angio-associated migratory cell protein (AAMP) was first isolated by Beckner et al. in 1995 from a human melanoma cell line (Beckner et al., 1995), with histological analyses having revealed it to be expressed at high levels in cytotrophoblasts, endothelial cells, and poorly differentiated colon adenocarcinoma cells (Beckner et al., 1995). More recent research has highlighted an important role for AAMP as a regulator of the functionality of endothelial, smooth muscle, non-small cell lung cancer, and breast cancer cells (Holvoet and Sinnaeve, 2008;Vogt et al., 2008;Bielig et al., 2009; Yin et al., 2013;Hu et al., 2016;Yao et al., 2019;Yao et al., 2021). Intracellularly, AAMP is primarily observed within the cytoplasm and in association with the plasma membrane, in addition to being secreted into the outer membrane matrix (Beckner et al., 1995). This diversity of intercellular localization results suggests that AAMP may be involved in a wide array of cellular functions. Bielig et al. determined that AAMP can participate in HEK293T cell signal transduction (Bielig et al., 2009), while other researchers have demonstrated that it can activate RhoA/ROCK signaling to promote the mobilization of smooth muscle cells and the migration of vascular endothelial cells, thereby contributing to atherosclerotic and angiogenic processes (Holvoet and Sinnaeve, 2008; Hu et al., 2016). AAMP has further been reported to promote proliferation, metastasis, and chemoresistance in breast and non-small cell lung cancer (Yin et al., 2013;Yao et al., 2019; Yao et al., 2021). A negative relationship between AAMP upregulation and poor breast cancer patient clinical outcomes has also been reported (Yin et al., 2013). How AAMP functions in OS, however, has not been described to date.

Yes-associated protein (YAP) is a transcriptional coactivator that functions as a downstream signaling mediator within the Hippo signaling pathway(Zanconato et al., 2019), playing critical roles in the context of tissue regeneration and organ development (Moya and Halder, 2019). YAP signaling can contribute to many malignant phenotypes such as enhanced proliferation, the loss of polarity, the acquisition of chemoresistance, and tumor metastasis (Zanconato et al., 2019). Indeed, researchers had demonstrated the ability of YAP to promote metastasis and mobility in gastric cancer, liver cancer, and clear cell renal cell carcinoma (Qiao et al., 2017; Shi et al., 2018;Kim et al., 2021). The interplay between AAMP and YAP in the context of OS metastasis, however, has not been explored in detail to date.

Mitochondria function as mediators of key intracellular processes including signal transduction, ATP production, reactive oxygen species (ROS) generation, and the maintenance of calcium homeostasis, and are regulated by dynamic processes including fission, fusion, and mitophagy (Genovese et al., 2021). At the cellular level, these organelles influence growth, differentiation, signaling, and apoptotic cell death ( $\mathrm{Ji}$ et al., 2021), serving as the primary source of ATP, which is the de facto currency of cellular energetics (Foo et al., 2021). Localized mitochondrial ATP generation is leveraged in the context of invasion and motility through focal adhesion turnover (Scheid et al., 2021), with mitochondrial metabolic outputs from 
the glycolysis and oxidative phosphorylation pathways influencing such motility (Scheid et al., 2021). Mitochondrial fission and fusion have been reported to shape oncogenic processes in breast, endometrial, and liver cancer (Guo et al., 2020; Hu et al., 2020;Li et al., 2020). However, how mitochondrial functionality is tied to OS metastatic progression has not been clarified to date.

Herein, we employed a series of clinical, bioinformatics, in vitro, and in vivo assays that ultimately revealed AAMP to be highly upregulated in OS patients with pulmonary metastases and to be correlated with a poor prognosis. Moreover, AAMP was found to regulate YAP and to thereby shape OS cell migratory and invasive activity, while the knockdown of AAMP resulted in mitochondrial dysfunction and thus inhibited the malignant invasion and migration of these tumor cells.

\section{Materials And Methods OS patient samples}

All tissue samples were collected from patients at the Chongqing University Three Gorges Hospital who had provided written informed consent. In total, 5 pairs of primary OS tumor and lung metastatic OS samples were collected. The ethics committee of Chongqing University Three Gorges Hospital approved the present study. TARGET-OS RNA-seq and survival data were downloaded from the XENA Open Network database (https://xena.ucsc.edu), while the GSE21257 sequencing dataset and corresponding clinical data were downloaded from the GEO database (https://www.ncbi.nlm.nih.gov/gds/). Analyses of patient overall and metastasis-free survival were conducted using R ( $v$ 4.1.0) with the survival (v 3.1-12) and survminer ( $\mathrm{v}$ 0.4.8) packages. The Tumor OS public dataset (R2: Genomics Analysis and Visualization Platform (amc.nl). Accessed 4 Jan 2021), consisting of 127 OS samples, was utilized for result validation. The R2 platform (http://r2.amc.nl. Accessed 4 Jan 2021) was used for microarray data analyses

\section{Immunohistochemistry}

Immunohistochemical (IHC) staining was performed by fixing samples using $4 \%$ paraformaldehyde, embedding them in paraffin, and using a microtome to cut them into $4 \mu \mathrm{m}$-thick serial sections which were then blocked, probed with primary antibodies (1:500) (Additional file 1: Table S1), and imaged with a microscope. Two experienced pathologists independently reviewed the stained tissues. The percentage of positive staining and staining intensity were recorded for each sample, then immunoreactivity score (IRS) was valued by multiplying positive staining percentage with staining intensity. The expression of AAMP was defined according to the median IRS.

\section{Cell lines}

The U2OS, SAOS2, MG63, and 143B human OS cell lines were obtained from the Chinese Academy of Sciences Cell Bank. All cells were cultured in DMEM (Gibco) containing 10\% FBS and penicillin/streptomycin in a $37^{\circ} \mathrm{C} 5 \mathrm{CO}_{2}$ incubator. 


\section{Plasmid and vector construction}

The AAMP DNA fragment was amplified via PCR and inserted into the pCDH-vector (see Additional file 1: Table S1 for PCR primers). shRNA constructs specific for AAMP (shAAMP-1 and shAAMP-2) were synthesized by Tsingke (Beijing, China) and inserted into the pLKO.1 vector (see Additional file 1: Table S2 for shRNA sequences). Lentiviral knockdown or overexpression of YAP was conducted as in our previous studies(Zhan et al., 2021).

\section{Western blotting}

RIPA buffer (Beyotime, Beijing, China) was used to prepare protein lysates from cells, after which the concentrations of those proteins were determined using a BCA kit (Beyotime, Beijing, China). Samples of equal size $(30 \mu \mathrm{g})$ were then separated via SDS-PAGE and transferred onto PVDF membranes (Millipore) that were incubated overnight with appropriate primary antibodies (Additional file 1: Table S3) at $4^{\circ} \mathrm{C}$, followed by incubation with a secondary antibody for $1 \mathrm{~h}$. Protein bands were then detected using a high sensitivity electrochemiluminescence detection kit and a chemiluminescence imaging system (Bio-Rad, USA), with ImageJ being used for densitometric analyses.

\section{Transwell assays}

OS cells $\left(6 \times 10^{4}\right)$ were added to the upper chamber of a Transwell insert ( $8 \mu \mathrm{m}$ pore size; Corning, USA). For analyses of cellular invasion, this chamber was coated with Matrigel prior to cell addition. Cells were incubated in these chambers for $24 \mathrm{~h}$, after which migratory or invasive cells were fixed using $4 \%$ paraformaldehyde, stained with $0.1 \%$ crystal violet (Beyotime), and imaged in five fields of view per well with a light microscope (Olympus).

\section{Wound healing assay}

Cells were added to 6 -well plates $\left(4 \times 10^{5} /\right.$ well) until confluent, at which time a scratch wound was generated in the monolayer surface using a sterile $200 \mu \mathrm{l}$ pipette tip. The wound area was imaged at 0 and $24 \mathrm{~h}$ post-wounding, with cell migration then being imaged via inverted light microscope, and wound width being quantified using ImageJ.

\section{F-actin staining and filopodia analyses}

Cells were plated onto adhesive microscope slides ( $1 \times 10^{3} /$ well) and grown in DMEM containing $10 \%$ FBS for $24 \mathrm{~h}$, after which they were rinsed with PBS and fixed with $3.7 \%$ paraformaldehyde for $20 \mathrm{~min}$. After three additional washes using PBS supplemented with $0.1 \%$ Triton X-100, F-actin staining was performed for $1 \mathrm{~h}$ using Actin-Tracker Green-phalloidin (Cat. C2201S, Beyotime) in the dark at room temperature, followed by nuclear counterstaining using DAPI (Cat.C1005, Beyotime). Cells were then imaged via scanning microscope (Nikon, Japan) at 600x magnification. This same approach was used for MG63 cells overexpressing AAMP after $24 \mathrm{~h}$. ImageJ was used for filopodia quantification. 


\section{Transmission electron microscopy (TEM)}

143B and MG63 cells were plated overnight in Petri dishes $\left(1 \times 10^{6}\right.$ cells/plate). After a $24 \mathrm{~h}$ incubation in standard culture medium, cells were harvested, fixed using 2.5\% glutaraldehyde and $1 \%$ osmic acid, dehydrated with an ethanol gradient and acetone, embedded, cut into ultra-thin sections, stained using 3\% uranyl acetate-lead citrate, and imaged via TEM (JEM-1400 Plus, JEOL, Japan).

\section{Measurements of cellular ATP}

An ATP Assay Kit (ab83355, Abcam) was used to measure ATP levels in samples using reagents prepared based on provided protocols. Initially, $50 \mu \mathrm{L}$ ATP standards were prepared for use as a standard curve, after which $1 \times 10^{6}$ cells in 6-well plates were lysed on ice using lyolysis. Samples were then centrifuged for $5 \mathrm{~min}$ at $13,000 \times \mathrm{g}$ at $4^{\circ} \mathrm{C}$, and supernatants were collected for analysis. All samples were adjusted to a final volume of $50 \mu \mathrm{L}$ using ATP Assay Buffer, with $50 \mu \mathrm{L}$ of Reaction Mix being added to each standard and sample well, and $50 \mu \mathrm{L}$ of Background Reaction Mix being added to background control wells. After a 30 min incubation in the dark at room temperature, absorbance was assessed at $570 \mathrm{~nm}$ via microplate reader. All analyses were repeated in triplicate.

In vivo pulmonary metastasis model

To explore the in vivo impact of knocking down AAMP on the metastasis of OS cells to the lung, BALB/c nude mice (5-weeks-old; Hunan Laike Jingda Experimental Animal Co. LTD, China) were randomized into shNC and shAAMP-1 groups that were injected via the tail vein with $5 \times 10^{6} 143 \mathrm{~B}$ - shNC or shAAMP-1 cells. Murine body weight was measured every third day, and on day 30 post-injection, mice were euthanized. Lung tissues were then collected, fixed with 4\% paraformaldehyde, paraffin-embedded, cut into sections, and stained with hematoxylin and eosin (H\&E). Metastatic nodules were then visualized and counted via light microscopy.

\section{Statistical analyses}

Data were compared via Student's t-tests or one-way ANOVAs, and all experiments were conducted a minimum of three times. $P<0.05$ was the threshold of significance unless otherwise noted. SPSS 26.0 (IL, USA) or GraphPad Prism 8.00 (CA, USA) were sued for all statistical analyses.

\section{Results}

\section{Patients with metastatic OS metastasis exhibit AAMP that correlates with a poor patient prognosis}

While AAMP has been reported to function in an oncogenic manner in breast and lung cancer (Yin et al., 2013;Yao et al., 2021), its role in OS has yet to be characterized. Herein, we began by comparing the association between AAMP expression levels and the odds of overall survival among 84 OS patients in 
the GSE21257 dataset published previously. In this analysis, the overall survival of 32 patients exhibiting high levels of AAMP expression was significantly reduced relative to that of the 52 patients exhibiting low levels of AAMP expression (Fig. 1a). We then assessed AAMP expression levels in the GSE124768 dataset consisting of OS and recurrent OS xenograft samples using an online bioinformatics platform (http://r2.amc.nl. Accessed 4 Jan 2021), revealing AAMP expression to be increased in recurrent OS relative to primary OS samples (Fig. 1b). AAMP expression in OS samples varied as a function of the tissue being assessed (tibia, right humerus, femur, right distal femur, and lung mass), with the highest levels being evident in analyzed metastatic lung masses (Fig. 1C). Consistently, IHC staining of 5 pairs of primary OS and lung metastatic OS tumor tissue samples revealed significantly increased AAMP expression in metastatic lung nodules from these patients (Fig. 1d). Together, these results suggest that AAMP may function as an important driver of OS pulmonary metastasis.

To further expand on these findings, Western blotting was used to assess AAMP levels in four human OS cell lines (U2OS, SAOS2, 143B, MG63), revealing variable levels of this protein among cell lines (Fig. 1e, f). The MG63 and 143B cell lines, which exhibited the highest levels of AAMP expression, we used in subsequent follow-up assays, and were employed to generate cells in which AAMP had been stably knocked down via lentiviral transduction as confirmed via Western blotting (Fig. 1g, h).

\section{Knocking down AAMP suppresses OS cell migratory, invasive, and epithelial-mesenchymal transition (EMT) activity}

To better understand how AAMP influences OS cell migratory and invasive activity, wound healing and Transwell assays were conducted using MG63 and 143B cells in which AAMP had been knocked down. Significant reductions in the migration and invasion of 143B-shAAMP and MG63-shAAMP cells were observed relative to corresponding controls (Fig. 2a-d). The EMT is a critical process that is integral to the pulmonary metastatic progression of OS and other cancer types (Han et al., 2019;Lin and Wu, 2020). Western blotting was thus used to assess the expression of EMT-associated marker proteins in 143B and MG63 cells following AAMP knockdown. In cells in which AAMP had been knocked down, the epithelial marker E-cadherin was upregulated, with a concomitant drop in the expression of the mesenchymal markers N-cadherin and Vimentin (Fig. 2e). In addition, MMP-9 and Snail were downregulated in cells in which AAMP had been knocked down relative to corresponding controls. These data thus indicate that AAMP knockdown can disrupt OS cell migratory and invasive activity in part via the inhibition of the EMT.

\section{AAMP regulates CFL1 to promote OS cell protrusion}

Cofilin-1 (CFL1) can promote the reorganization of the actin cytoskeleton and EMT induction in colorectal cancer cells(Sousa-Squiavinato et al., 2019), and has also been proposed to be a mediator of chemoresistance and metastasis in prostate cancer (Chen et al., 2020). Bioinformatics analyses of 53 OS patient samples (R2: Two Gene View for AAMP with CFL1 (amc.nl) Accessed 4 Jan 2021) revealed AAMP and CFL1 gene expression levels to be positively correlated (Fig. 3a), and Western blotting revealed p- 
CFL1 levels to be significantly reduced following the knockdown of AAMP (Fig. 3b, c). Such p-CFL1 is important for maintaining F-actin stability (Qiao et al., 2017). Lamellipodium formation is critical to the movement of cancer cells, and as these lamellipodia are composed of F-actin, the degradation of F-actin can result in lamellipodium collapse (Shi et al., 2018). Given our above findings, we speculated that AAMP may drive OS cell migratory by promoting lamellipodium formation. We thus utilized phalloidin to stain for F-actin in MG63 and 143B cells in which AAMP had or had not been knocked down. These assays revealed that AAMP knockdown cells exhibited a smoother cell surface relative to control cells (Fig. 3d, e), with fewer evident protrusions (red arrows) consistent with the inhibition of lamellipodium formation. AAMP overexpression, in contrast, was sufficient to enhance lamellipodium formation in OS cells (Fig. 3f). These data suggest that AAMP can regulate OS cell lamellipodium formation at least in part by regulating $\mathrm{p}$-CFL1 levels within cells, thereby enhancing their migratory and invasive potential.

\section{Knocking down AAMP induced mitochondrial dysfunction and impairs ATP production in OS cells}

Mitochondria are key mediators of cellular energy production in the form of ATP (Foo et al., 2021), influencing proliferative activity, apoptosis, and differentiation while also contributing to ROS generation, regulating calcium homeostasis, and coordinating signal transduction (Foo et al., 2021;Ji et al., 2021). Tumor cells require large quantities of ATP to move effectively (Garde and Sherwood, 2021;Scheid et al., 2021). Both F-actin and CFL1 have recently been shown to serve as important regulators of mitochondrial functionality (Rehklau et al., 2017; Hoffmann et al., 2019), and reductions in F-actin expression and CFL1 phosphorylation can suppress OS cell migratory and invasive activity (Hsieh et al., 2021). In light of our above results, we speculated that AAMP may regulate mitochondrial dynamics within OS cells.

Consistently, TEM analyses revealed that AAMP knockdown was associated with the swelling and vacuolation of mitochondria in MG63 and 143B cells with a concomitant loss of mitochondrial cristae (red arrows), whereas no corresponding disruption of mitochondrial morphology was evident in control cells (green arrow, Fig. 4a). Western blotting revealed that AAMP knockdown was associated with increases in the expression of the mitochondrial fission markers MFF and p-DRP1(S616) relative to control cells, with a corresponding drop in the levels of the mitochondrial fusion marker proteins OPA1 and MFN2 (Fig. 4b-d). ATP levels were also found to be significantly reduced in AAMP-knockdown OS cells relative to corresponding controls (Fig. 4e). These data thus suggest that the knockdown of AAMP within OS cells can result in aberrant mitochondrial functionality and impaired ATP synthesis.

\section{Knocking down AAMP suppresses the migratory and invasive activity of OS cells by inhibiting YAP}

We have previously shown YAP to function as a promoter of OS cell resistance to photodynamic therapy owing to its ability to protect against apoptotic cell death (Zhan et al., 2021), and other studies have further shown that YAP can enhance the migratory and invasive activity of a range of cancer cell types (Yamaguchi and Taouk, 2020;Barrette et al., 2021;Sun et al., 2021), including OS cells (Kovar et al., 2020). 
An initial bioinformatics analysis revealed the expression of AAMP and YAP to be positively correlated in OS patients (R2: Two Gene View for AAMP with YAP1 (amc.nl) Accessed 4 Jan 2021)(Fig. 5a), and patients exhibiting higher levels of YAP expression exhibited reduced metastasis-free survival rates (R2: Kaplan Meier Scanner (amc.nl) Accessed 4 Jan 2021)(Fig. 5b). The gene expression level of YAP in metastatic osteosarcoma was significantly higher than that in patients without metastasis (R2: Two Gene View for NG_5yrmetastases with YAP1 (amc.nl).Accessed 4 Jan 2021)(Fig. 5c).To expand on these analyses, we stably knocked down or overexpressed YAP in the MG63 and 143B cell lines, and we confirmed successful knockdown or overexpression via Western blotting (Fig. $5 \mathrm{~d}-\mathrm{g}$ ). Transwell assays revealed that the migration of both of these cell lines was significantly suppressed following YAP knockdown relative to control cells (Fig. 5h, i), whereas YAP overexpression enhanced both migratory and invasive activity for these cell lines (Fig. 5h, i). Western blotting further revealed that the knockdown of AAMP in both MG63 and 143B cells was associated with decreases in the levels of YAP and its downstream targets CTGF and CYR61 relative to control cells (Fig. 5j, k). We then assessed the levels of EMT-related marker proteins in these cells, revealing YAP knockdown to result in the upregulation and downregulation of epithelial and mesenchymal marker proteins, respectively (Fig. 5l-n). These data suggest that AAMP can regulate YAP to promote OS cell invasion, migration, and EMT induction.

\section{Knocking down AAMP suppresses the in vivo metastasis of OS cells}

To further confirm the ability of AAMP to contribute to the pulmonary metastasis of OS, we implemented a model system in which nude mice received an intravenous injection of 143B cells in which AAMP had or had not been stably knocked down. While there were no differences in murine body weight when comparing the shNC and shAAMP-1 groups (Fig. 6a), metastatic lung nodules were evident in 4/5 mice in the shNC group but in only $1 / 5$ mice in the shAAMP-1 group (1/5) (Fig. 6b, c). There were also significantly more metastatic nodules in the lungs of mice in the shNC group relative to those of mice in the shAAMP group (Fig. 6d). In summary, the knockdown of AAMP can suppress the in vivo metastasis of OS cells to the lungs.

\section{Discussion}

Previous reports suggest that AAMP can function in part by promoting migratory activity in vascular endothelial, smooth muscle, breast, and non-small cell lung cancer cells (Holvoet and Sinnaeve, 2008;Vogt et al., 2008;Yin et al., 2013;Hu et al., 2016;Yao et al., 2021). The overexpression of AAMP results in enhanced migratory activity in vascular smooth muscle cells, while antibody or siRNA transfection mediated inhibition of AAMP can reverse this effect (Holvoet and Sinnaeve, 2008). AAMP downregulation results in decreases in RhoA activity levels within vascular smooth muscle cell membrane components (Holvoet and Sinnaeve, 2008;Vogt et al., 2008), consistent with the ability of this protein to regulate angiogenesis and migration activity in vascular endothelial cells by controlling RhoA activation (Hu et al., 2016). We have previously reported that reductions in RhoA activity can suppress 
YAP activity via the RhoA/ ROCK2/LIMK2/CFL1 pathway, thereby compromising the ability of OS cells to tolerate photodynamic therapy (Zhan et al., 2021). AAMP promotes proliferative activity and chemoresistance within NSCLC cells through interactions with epidermal growth factor (Yao et al., 2019), and it has also been suggested to interact with CDC42 within NSCLC cells to drive their invasive and migratory activity (Yao et al., 2021). Herein, we found that OS patients exhibiting high levels of AAMP expression exhibited a worse prognosis than patients expressing lower levels of this protein, with such upregulation being more pronounced in patients with recurrent or lung metastatic $O S$ relative to patients with primary OS. The knockdown of AAMP significantly suppressed OS cell migratory and invasive activity in addition to blunting EMT induction. Given the central role of the EMT in tumor metastatic progression (Lin and Wu, 2020), we posit that AAMP may function as a key regulator of OS cell metastasis.

The terminal Rho GTPase signaling cascade effector protein CFL1 is an important regulator of actin dynamics within cells, functioning by cutting F-actin and promoting filament depolymerization (SousaSquiavinato et al., 2019). The overexpression of CFL1 has been reported in many tumors wherein it regulates cytoskeletal recombination, EMT induction, and lamellipodium formation (Xu et al., 2021). The inactivation of CFL1 is mediated by its Ser3 phosphorylation by LIMK1/LIMK2 and by upstream Rho GTPase signaling intermediates such as Rac1, RhoA, and Cdc42 (Xu et al., 2021). The phosphorylation of CFL1 causes it to lose its affinity for actin, leading to the inhibition of depolymerization and cleavage, while its phosphatase-mediated dephosphorylation results in CFL1 activation. AAMP is an important upstream regulator of RhoA activation (Holvoet and Sinnaeve, 2008;Vogt et al., 2008;Hu et al., 2016). Herein, we found AAMP and CFL1 expression levels to be positively correlated in OS patients. Following AAMP knockdown, p-CFL1 and F-actin levels declined, and lamellipodia formation was reduced. These data align well with NSCLC-related findings published by Yao et al. (Yao et al., 2021). As such, we hypothesized that AAMP may control OS cell migration and invasion by regulating RhoA activity and influencing cytoskeletal dynamics via the RhoA /ROCK/LIMK/CFL1 axis.

The mitochondrial are key coordinators of cellular metabolic activity, generating ATP through the TCA cycle and oxidative phosphorylation in addition to regulating diverse cellular processes(Foo et al., 2021; Ji et al., 2021). Tumor cell invasion/migration and associated F-actin polymerization consume large quantities of ATP (Garde and Sherwood, 2021). CFL1 has previously been shown to maintain mitochondrial morphology and to regulate mitochondrial dynamics (Rehklau et al., 2017;Hoffmann et al., 2019). When activated, CFL1 is transported to the mitochondrial outer membrane where it is able to interact with dynamin-related protein 1 (Drp1), inducing mitochondrial fission, cytochrome $C$ release, and consequent tumor cell apoptosis (Hoffmann et al., 2019; Hu et al., 2020). CFL1 can further regulate EMT induction within colorectal cancer (CRC) cells by promoting cytoskeletal rearrangement and cell-cell interactions (Sousa-Squiavinato et al., 2019). Herein, we found that the knockdown of AAMP was associated with mitochondrial swelling, a loss of mitochondrial cristae, impaired mitochondrial fusion, and reduced ATP production, thereby inhibiting OS cell migration and invasion. CFL1 has recently been suggested to function as a regulator of chemoresistance and metastasis in prostate cancer (Chen et al., 2020). In metastatic HCC, Zhang et al. have suggested that excessive mitochondrial fission is 
predominant (Zhang et al., 2020), which may have important research implications. Moreover, Chuang et al. (Chuang et al., 2020) have reported that ROS generation can disrupt normal mitochondrial dynamics, resulting in increased fission, autophagic activity, and mitochondrial dysfunction within skin cancer cells. These results suggest that AAMP regulates CFL1 phosphorylation, resulting in changes in mitochondrial morphology, function, and ATP production that ultimately shape the migration and invasion of OS cells (Fig. 6e).

YAP is a transcriptional coactivator that functions as a downstream component of the Hippo signaling pathway (Zanconato et al., 2019), and it is an important mediator of organ development and tissue regeneration(Moya and Halder, 2019). Prolonged, excessive YAP activation is a common hallmark of oncogenesis (Moya and Halder, 2019). Mechanistically, Shi et al. (Shi et al., 2018) found that YAP was able to regulate the CFL/F-actin/lamellipodium axis to drive metastatic activity in hepatocellular carcinoma (HCC) cells. Qiao et al. (Qiao et al., 2017) further reported YAP to regulate ARHGAP29 within gastric cancer cells and to thereby control F-actin dynamics to regulate metastatic progression. AAMP has previously been shown to regulate RhoA activity within vascular endothelial and smooth muscle cells (Holvoet and Sinnaeve, 2008;Vogt et al., 2008; Hu et al., 2016). Our prior work suggests that reductions in RhoA activity within OS cells can inhibit the activity of YAP via a RhoA/ROCK2/LIMK2/CFL1 signaling axis (Zhan et al., 2021). We thus hypothesized that AAMP may be able to regulate YAP to control the migratory and invasive activity of OS cells. To test this hypothesis, we conducted bioinformatics analyses which revealed YAP and AAMP expression levels to be strongly positively correlated in OS. Patients with high levels of YAP expression exhibited significantly lower rates of metastasis-free survival relative to patients with lower levels of YAP expression. Knocking down YAP was sufficient to reduce the migratory and invasive activity of OS cells in vitro, whereas its overexpression had the opposite effect. YAP protein levels and those of downstream target genes also fell significantly following the knockdown of AAMP, together with reductions in EMT marker protein expression. These data are consistent with recent evidence published by Morice et al. (Morice et al., 2021), who demonstrated that YAP can bind to Smad3 in response to TGF-B signaling, thereby promoting OS pulmonary metastasis. Overall, these data suggest that AAMP can promote OS invasion and migration at least in part by enhancing YAP activation.

To further confirm the functional importance of AAMP as a mediator of OS pulmonary metastasis, we established a murine model of lung metastasis. In this experimental context, AAMP knockdown significantly reduced the number of metastatic lung nodules observed in mice without any concomitant reduction in murine body weight, potentially as a consequence of the selected experimental time point. These results thus suggest that AAMP is an important mediator of OS cell metastasis, and that the therapeutic targeting of AAMP may be a viable approach to suppressing the metastatic progression of this form of cancer.

There are certain limitations to the present study. For one, no large-scale analyses of clinical samples were performed, although corresponding analyses of a limited subset of primary tumors and lung metastases were conducted along with supporting bioinformatics research. Secondly, no rescue experiments associated with YAP or AAMP were conducted, although the overexpression and knockdown 
of these genes were evaluated in two cell lines, and the impact of AAMP on OS cell migratory and invasive activity was assessed in the context of AAMP knockdown-associated mitochondrial dysfunction, with animal studies further being used to validate these results.

\section{Conclusions}

In conclusion, our data indicate that AAMP can promote OS cell migration and invasion at least in part by regulating YAP and mitochondrial functionality. As such, targeting the AAMP/CFL1/YAP signaling axis may be of value as a novel approach to preventing the metastatic progression of OS.

\section{Abbreviations}

AAMP

Angio-associated migratory cell protein

YAP

Yes-associated protein

OS

Osteosarcoma

$\mathrm{IHC}$

Immunohistochemical

EMT

Epithelial-mesenchymal transition

TEM

Transmission electron microscopy

CFL1

Cofilin1

ROS

Reactive oxygen species

IRS

Immunoreactivity score

FBS

Fetal bovine serum

DMEM

Dulbecco's modified Eagle medium

H\&E

Hematoxylin and eosin

NSCLC

Non-small-cell lung cancer

$\mathrm{CRC}$

Colorectal cancer 
HCC

Hepatocellular Carcinoma

\section{Declarations}

\section{Authors' contributions}

XLX, CJ and OYS contributed to the conception and experimental design of the study. ZFB and CZY performed the biological experiments, analyzed the data, and prepared the figures and tables. ZFB and XCZ wrote and edited the manuscript. DQR provided the reagents and advice on the overall project. XLX provided the overall project leadership. XLX, CZY, XCZ, DQR, JCC, LHH, TL, XS, XJX, ZFB, CJ and OYS discussed the results. All authors read and approved the final manuscript.

\section{Funding}

This work was supported in part by The National Natural Science Foundation of China grants (81572634, 82172682); the General project of Chongqing Natural Science Foundation (cstc2019jcyj-msxmX0358, cstc2019jcyj-msxmX0801, cstc2020jcyj-msxmX0951, cstc2020jcyj-msxmX0541, cstc2021jcyjmsxmX0909), Chongqing medical scientific research project (Joint project of Chongqing Health Commission and Science and Technology Bureau) (2020FYYX087), Chongqing Yuzhong District basic research and frontier exploration project (2018114, 20200142), Graduate Education and Teaching reform research project of Chongqing University(cquyjg21305).

\section{Availability of data and materials}

All data generated or analyzed during this study are included in this published article.

\section{Ethics approval and consent to participate}

The collection of human specimens was performed in the accordance with the Ethical Committee of Human Research of the Chongqing University Three Gorges Hospital. Informed consent was obtained from all patients for being included in the study. The animal studies were approved by the Ethics Committee of the Chongqing University Three Gorges Hospital.

\section{Consent for publication}

Not applicable. 


\section{Competing interests}

The authors declare that they have no competing interests.

\section{References}

1. Bacci G, Rocca M, Salone M, Balladelli A, Ferrari S, Palmerini E, Forni C, Briccoli A. High grade osteosarcoma of the extremities with lung metastases at presentation: treatment with neoadjuvant chemotherapy and simultaneous resection of primary and metastatic lesions. J Surg Oncol. 2008;98:415-20.

2. Barrette AM, Ronk H, Joshi T, Mussa Z, Mehrotra M, Bouras A, Nudelman G, Raj J, Bozec JG, Lam D, Houldsworth W, Yong J, Zaslavsky R, Hadjipanayis E, Birtwistle CG, M.R., and Tsankova NM. (2021). Anti-invasive efficacy and survival benefit of the YAP-TEAD inhibitor Verteporfin in preclinical glioblastoma models. Neuro Oncol.

3. Beckner ME, Krutzsch HC, Stracke ML, Williams ST, Gallardo JA, Liotta LA. Identification of a new immunoglobulin superfamily protein expressed in blood vessels with a heparin-binding consensus sequence. Cancer Res. 1995;55:2140-9.

4. Belayneh R, Fourman MS, Bhogal S, Weiss KR. Update on Osteosarcoma. Curr Oncol Rep. 2021;23:71.

5. Bielig H, Zurek B, Kutsch A, Menning M, Philpott DJ, Sansonetti PJ, Kufer TA. A function for AAMP in Nod2-mediated NF-kappaB activation. Mol Immunol. 2009;46:2647-54.

6. Chen L, Cai J, Huang Y, Tan X, Guo Q, Lin X, Zhu C, Zeng X, Liu H, Wu X. (2020). Identification of cofilin-1 as a novel mediator for the metastatic potentials and chemoresistance of the prostate cancer cells. European Journal of Pharmacology.

7. Chuang KC, Chang CR, Chang SH, Huang SW, Chuang SM, Li ZY, Wang ST, Kao JK, Chen YJ, Shieh JJ. Imiquimod-induced ROS production disrupts the balance of mitochondrial dynamics and increases mitophagy in skin cancer cells. J Dermatol Sci. 2020;98:152-62.

8. Foo BJ, Eu JQ, Hirpara JL, Pervaiz S. (2021). Interplay between Mitochondrial Metabolism and Cellular Redox State Dictates Cancer Cell Survival. Oxid Med Cell Longev 2021, 1341604.

9. Garde A, Sherwood DR. (2021). Fueling Cell Invasion through Extracellular Matrix. Trends Cell Biol.

10. Genovese I, Carinci M, Modesti L, Aguiari G, Pinton P, Giorgi C. (2021). Mitochondria: Insights into Crucial Features to Overcome Cancer Chemoresistance. Int J Mol Sci 22.

11. Guo J, Ye F, Jiang X, Guo H, Xie W, Zhang Y, Sheng X. Drp1 mediates high glucose-induced mitochondrial dysfunction and epithelial-mesenchymal transition in endometrial cancer cells. Exp Cell Res. 2020;389:111880.

12. Han Y, Guo W, Ren T, Huang Y, Wang S, Liu K, Zheng B, Yang K, Zhang H, Liang X. Tumor-associated macrophages promote lung metastasis and induce epithelial-mesenchymal transition in osteosarcoma by activating the COX-2/STAT3 axis. Cancer Lett. 2019;440-441:116-25. 
13. Hoffmann L, Rust MB, Culmsee C. Actin(g) on mitochondria - a role for cofilin1 in neuronal cell death pathways. Biol Chem. 2019;400:1089-97.

14. Holvoet $P$, Sinnaeve P. Angio-associated migratory cell protein and smooth muscle cell migration in development of restenosis and atherosclerosis. J Am Coll Cardiol. 2008;52:312-4.

15. Hsieh YH, Hsu WH, Yang SF, Liu CJ, Lu KH, Wang PH, Lin RC. (2021). Potential Antimetastatic Effect of Timosaponin Alll against Human Osteosarcoma Cells through Regulating the Integrin/FAK/Cofilin Axis. Pharmaceuticals (Basel) 14.

16. Hu J, Qiu J, Zheng Y, Zhang T, Yin T, Xie X, Wang G. AAMP Regulates Endothelial Cell Migration and Angiogenesis Through RhoA/Rho Kinase Signaling. Ann Biomed Eng. 2016;44:1462-74.

17. Hu J, Zhang H, Li J, Jiang X, Zhang Y, Wu Q, Shen L, Shi J, Gao N. ROCK1 activation-mediated mitochondrial translocation of Drp1 and cofilin are required for arnidiol-induced mitochondrial fission and apoptosis. J Exp Clin Cancer Res. 2020;39:37.

18. Ji W, Tang X, Du W, Lu Y, Wang N, Wu Q, Wei W, Liu J, Yu H, Ma B, Li L, Huang W. (2021). Optical/electrochemical methods for detecting mitochondrial energy metabolism. Chem Soc Rev.

19. Kim N, Kim S, Lee MW, Jeon HJ, Ryu H, Kim JM, Lee HJ. (2021). MITF Promotes Cell Growth, Migration and Invasion in Clear Cell Renal Cell Carcinoma by Activating the RhoA/YAP Signal Pathway. Cancers (Basel) 13.

20. Kovar H, Bierbaumer L, Radic-Sarikas B. (2020). The YAP/TAZ Pathway in Osteogenesis and Bone Sarcoma Pathogenesis. Cells 9.

21. Li M, Wang L, Wang Y, Zhang S, Zhou G, Lieshout R, Ma B, Liu J, Qu C, Verstegen MMA, Sprengers D, Kwekkeboom J, Van Der Laan LJW, Cao W, Peppelenbosch MP, Pan Q. (2020). Mitochondrial Fusion Via OPA1 and MFN1 Supports Liver Tumor Cell Metabolism and Growth. Cells 9.

22. Lin YT, Wu KJ. Epigenetic regulation of epithelial-mesenchymal transition: focusing on hypoxia and TGF-beta signaling. J Biomed Sci. 2020;27:39.

23. Meltzer PS, Helman LJ. New Horizons in the Treatment of Osteosarcoma. N Engl J Med. 2021;385:2066-76.

24. Morice S, Danieau G, Tesfaye R, Mullard M, Brion R, Dupuy M, Ory B, Royer B-L, Corre B, Redini I, F., and Verrecchia F. (2021). Involvement of the TGF- $\beta$ Signaling Pathway in the Development of YAPDriven Osteosarcoma Lung Metastasis. Frontiers in Oncology 11.

25. Moya IM, Halder G. Hippo-YAP/TAZ signalling in organ regeneration and regenerative medicine. Nat Rev Mol Cell Biol. 2019;20:211-26.

26. Qiao Y, Chen J, Lim YB, Finch-Edmondson ML, Seshachalam VP, Qin L, Jiang T, Low BC, Singh H, Lim CT, Sudol M. YAP Regulates Actin Dynamics through ARHGAP29 and Promotes Metastasis. Cell Rep. 2017;19:1495-502.

27. Rehklau K, Hoffmann L, Gurniak CB, Ott M, Witke W, Scorrano L, Culmsee C, Rust MB. Cofilin1dependent actin dynamics control DRP1-mediated mitochondrial fission. Cell Death Dis. 2017;8:e3063-3. 
28. Scheid AD, Beadnell TC, Welch DR. Roles of mitochondria in the hallmarks of metastasis. $\mathrm{Br} \mathrm{J}$ Cancer. 2021;124:124-35.

29. Shi C, Cai Y, Li Y, Li Y, Hu N, Ma S, Hu S, Zhu P, Wang W, Zhou H. Yap promotes hepatocellular carcinoma metastasis and mobilization via governing cofilin/F-actin/lamellipodium axis by regulation of JNK/Bnip3/SERCA/CaMKII pathways. Redox Biol. 2018;14:59-71.

30. Smrke A, Anderson PM, Gulia A, Gennatas S, Huang PH, Jones RL. (2021). Future Directions in the Treatment of Osteosarcoma. Cells 10.

31. Sousa-Squiavinato ACM, Rocha MR, Barcellos-De-Souza P, De Souza WF, Morgado-Diaz JA. Cofilin-1 signaling mediates epithelial-mesenchymal transition by promoting actin cytoskeleton reorganization and cell-cell adhesion regulation in colorectal cancer cells. Biochim Biophys Acta Mol Cell Res. 2019;1866:418-29.

32. Sun B, Zhong FJ, Xu C, Li YM, Zhao YR, Cao MM, Yang LY. Programmed cell death 10 promotes metastasis and epithelial-mesenchymal transition of hepatocellular carcinoma via PP2Ac-mediated YAP activation. Cell Death Dis. 2021;12:849.

33. Vogt F, Zernecke A, Beckner M, Krott N, Bosserhoff AK, Hoffmann R, Zandvoort MA, Jahnke T, Kelm $\mathrm{M}$, Weber $\mathrm{C}$, Blindt R. Blockade of angio-associated migratory cell protein inhibits smooth muscle cell migration and neointima formation in accelerated atherosclerosis. J Am Coll Cardiol. 2008;52:30211.

34. Xu J, Huang Y, Zhao J, Wu L, Qi Q, Liu Y, Li G, Li J, Liu H, Wu H. Cofilin: A Promising Protein Implicated in Cancer Metastasis and Apoptosis. Front Cell Dev Biol. 2021;9:599065.

35. Yamaguchi H, Taouk GM. A Potential Role of YAP/TAZ in the Interplay Between Metastasis and Metabolic Alterations. Front Oncol. 2020;10:928.

36. Yao S, Shi F, Mu N, Li X, Ma G, Wang Y, Sun X, Liu X, Su L. Angio-associated migratory cell protein (AAMP) interacts with cell division cycle 42 (CDC42) and enhances migration and invasion in human non-small cell lung cancer cells. Cancer Lett. 2021;502:1-8.

37. Yao S, Shi F, Wang Y, Sun X, Sun W, Zhang Y, Liu X, Liu X, Su L. Angio-associated migratory cell protein interacts with epidermal growth factor receptor and enhances proliferation and drug resistance in human non-small cell lung cancer cells. Cell Signal. 2019;61:10-9.

38. Yin Y, Sanders AJ, Jiang WG. The impact of angio-associated migratory cell protein (AAMP) on breast cancer cells in vitro and its clinical significance. Anticancer Res. 2013;33:1499-509.

39. Zanconato F, Cordenonsi M, Piccolo S. YAP and TAZ: a signalling hub of the tumour microenvironment. Nat Rev Cancer. 2019;19:454-64.

40. Zhan F, He T, Chen Z, Zuo Q, Wang Y, Li Q, Zhong S, Ou Y. RhoA enhances osteosarcoma resistance to MPPa-PDT via the Hippo/YAP signaling pathway. Cell Biosci. 2021;11:179.

41. Zhang Z, Li TE, Chen M, Xu D, Zhu Y, Hu BY, Lin ZF, Pan JJ, Wang X, Wu C, Zheng Y, Lu L, Jia HL, Gao S, Dong QZ, Qin LX. MFN1-dependent alteration of mitochondrial dynamics drives hepatocellular carcinoma metastasis by glucose metabolic reprogramming. Br J Cancer. 2020;122:209-20. 
Figures

a

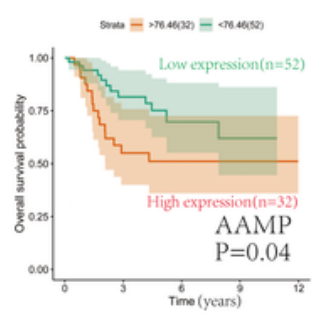

d
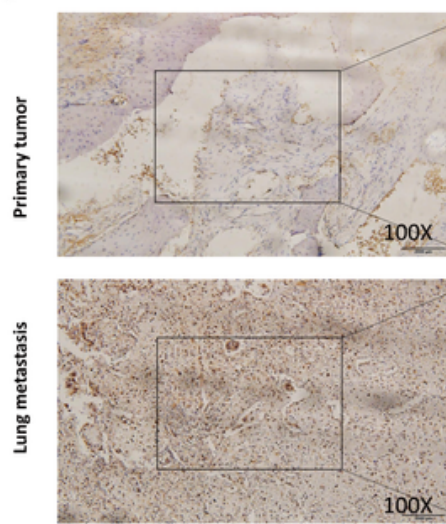

$100 x$
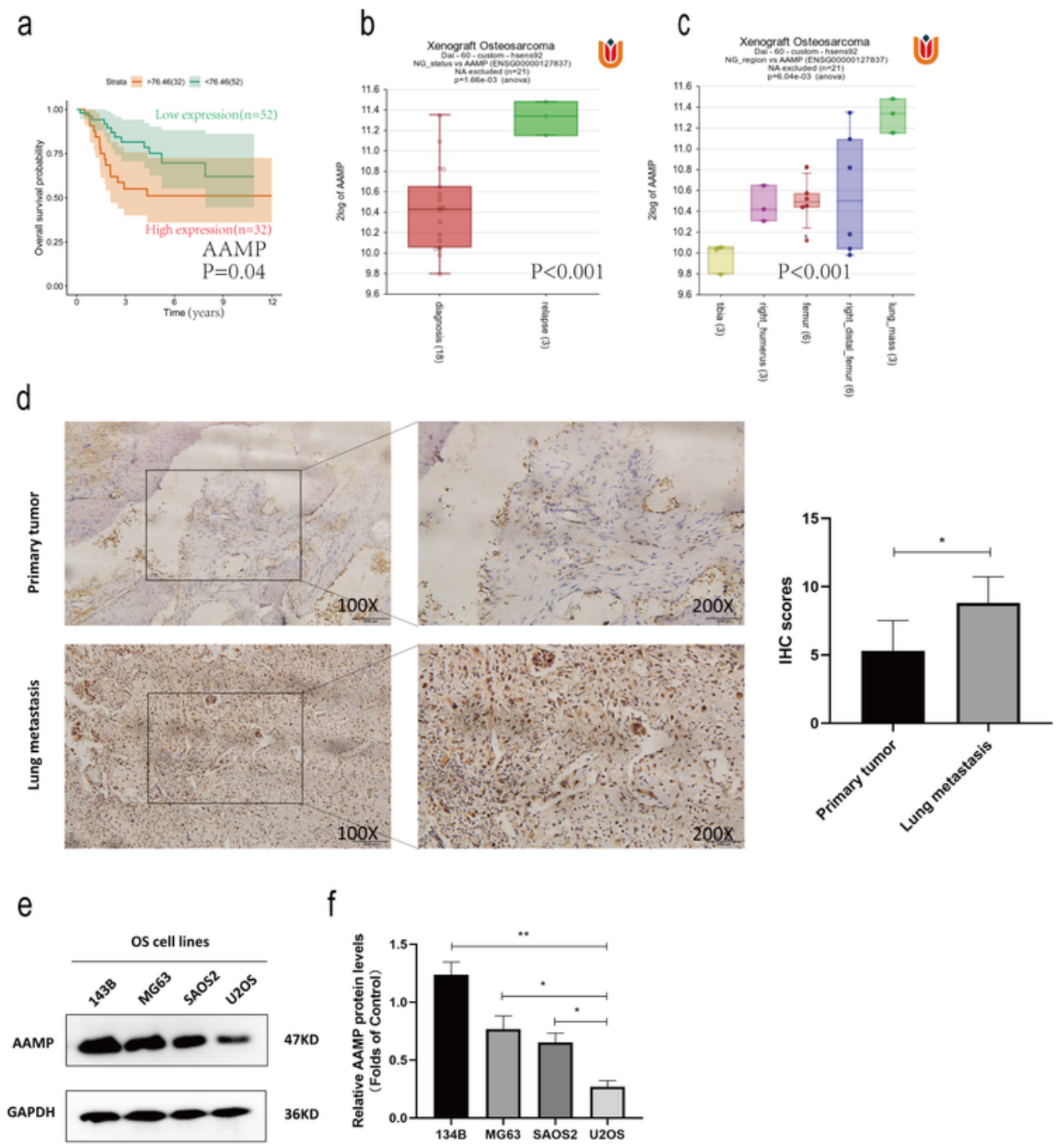

$f$

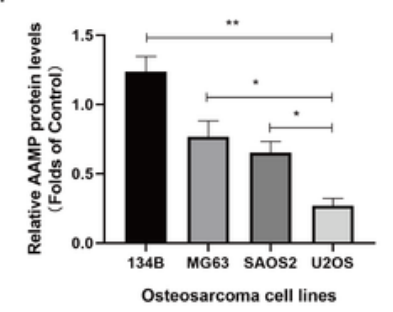

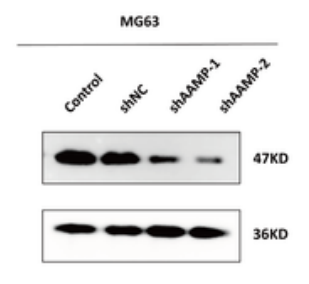

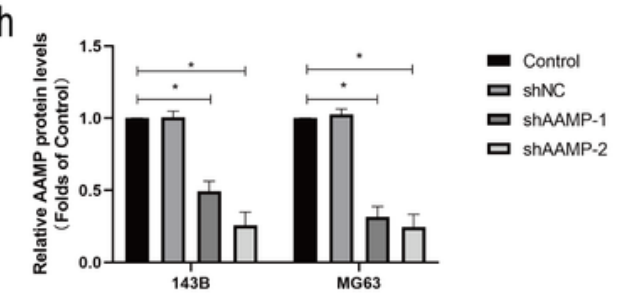

Figure 1

Patients with metastatic OS exhibit the upregulation of AAMP that is correlated with a poor prognosis. a Overall survival outcomes for 32 and 52 OS patients respectively exhibiting high and low levels of AAMP expression $(P<0.05)$. b AAMP levels were assessed in 18 OS xenograft samples and 3 recurrent OS 
samples $(P<0.001)$. c AAMP levels were measured in different portions of xenograft OS samples (tibia, right humerus, femur, right distal femur, and lung mass) ( $<$ 0.001). d AAMP expression in primary OS tissue samples and lung metastases was assessed via immunohistochemical staining. e, $f$ AAMP levels in OS cell lines (143B, MG63, SAOS2, and U2OS) were measured via Western blotting. $\mathbf{g}$, h AAMP levels were detected via Western blotting in 143B and MG63 levels following appropriate lentiviral transduction, with GAPDH as a loading control. All analyses were conducted in triplicate. ${ }^{*} P<0.05 * * P<0.01 * \star * P<$ 0.001

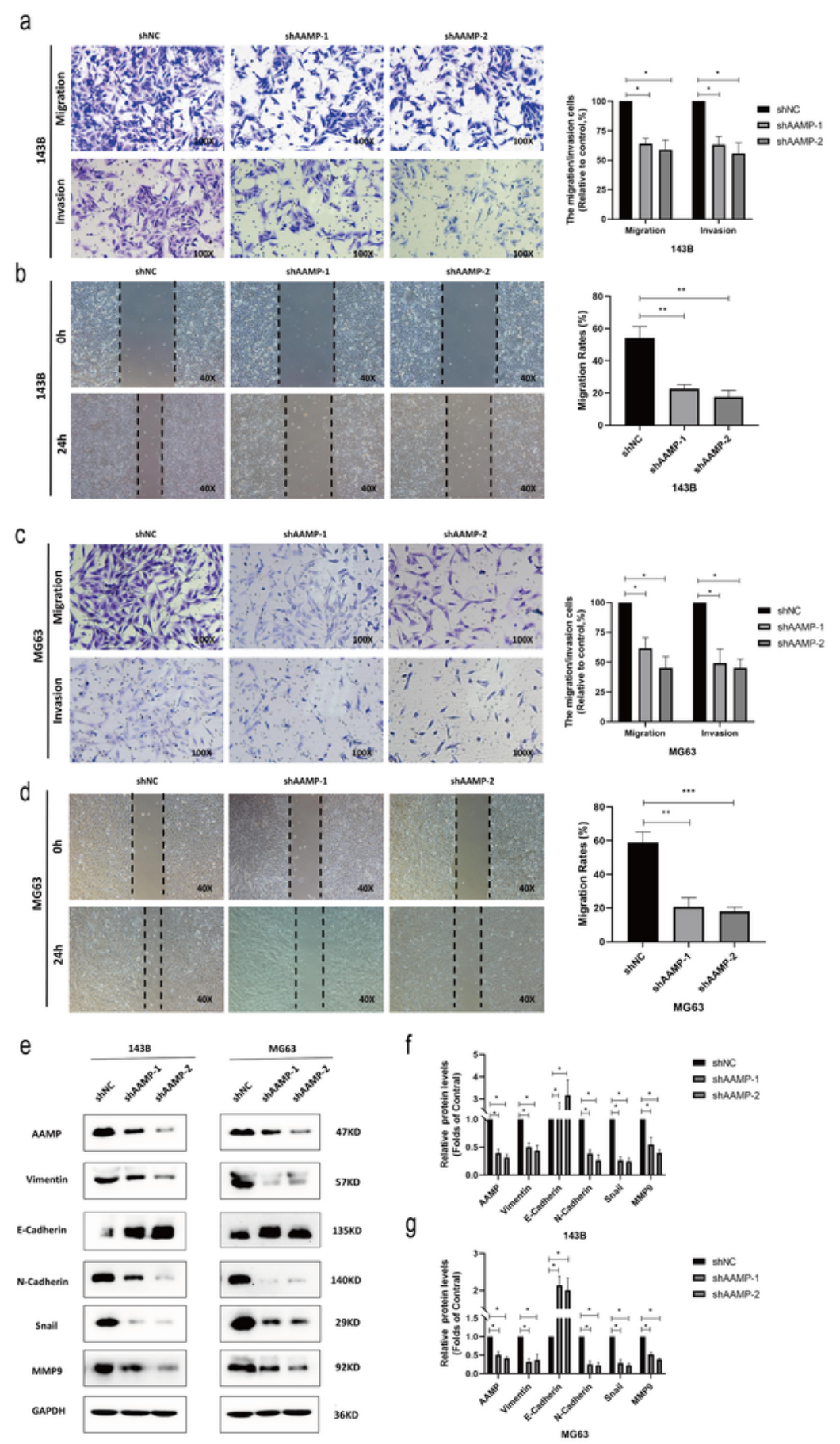


Figure 2

Knocking down AAMP suppresses the migratory, invasive, and epithelial-mesenchymal transition (EMT) activity of OS cells. a, b 143B cells were used in a wound healing assay following the knockdown of AAMP. c, d MG63 cells were used in a wound healing assay following the knockdown of AAMP. e-g EMTrelated protein levels were assessed via Western blotting after AAMP knockdown. All analyses were repeated in triplicate. ${ }^{*} \mathrm{P}<0.05^{*} \mathrm{P}<0.01{ }^{*} * \mathrm{P}<0.001$

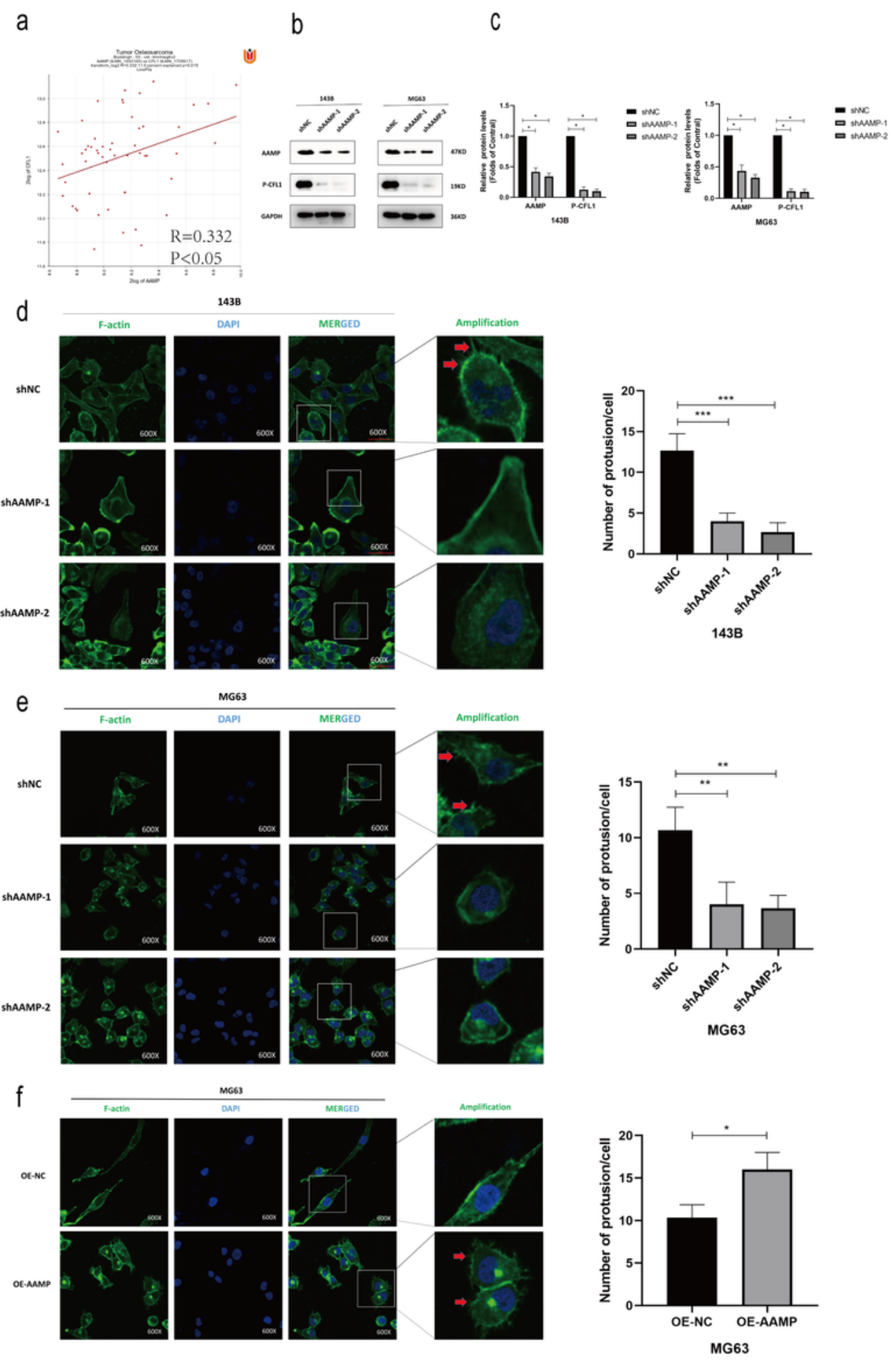


Figure 3

AAMP regulates CFL1 to promote OS cell protrusion. a A positive correlation between the expression of AAMP and CFL1 was evident in OS samples $(r=0.332, P<0.05)$. b, $\mathbf{c}$ Western blotting was used to assess p-CFL protein levels in OS cells following the knockdown of AAMP. d-f F-actin and nuclei were respectively stained using phalloidin (green) and DAPI (blue). Cells were imaged via laser confocal microscope, with protrusions being marked with red arrows. All analyses were repeated in triplicate. ${ }^{*} \mathrm{P}<$ $0.05 * * P<0.01 * * * P<0.001$ 
a
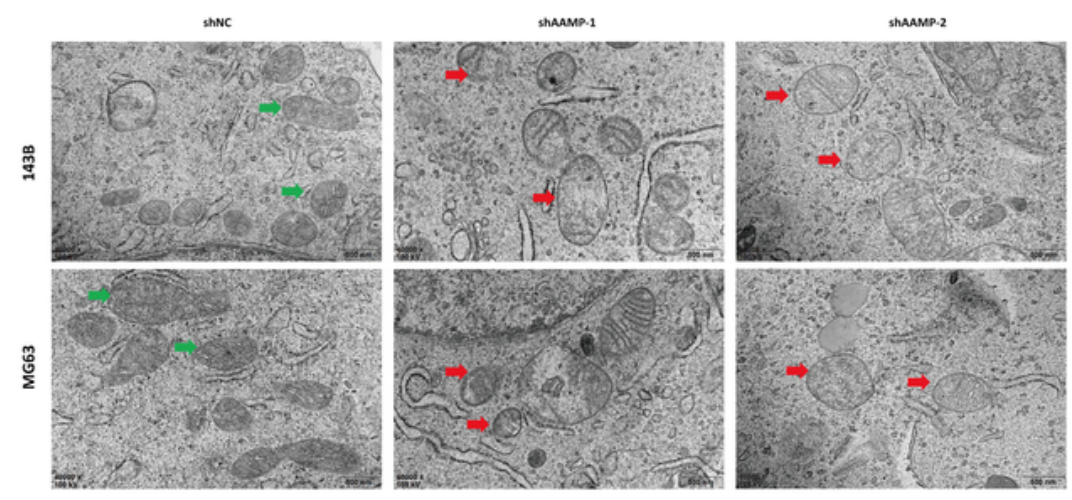

b

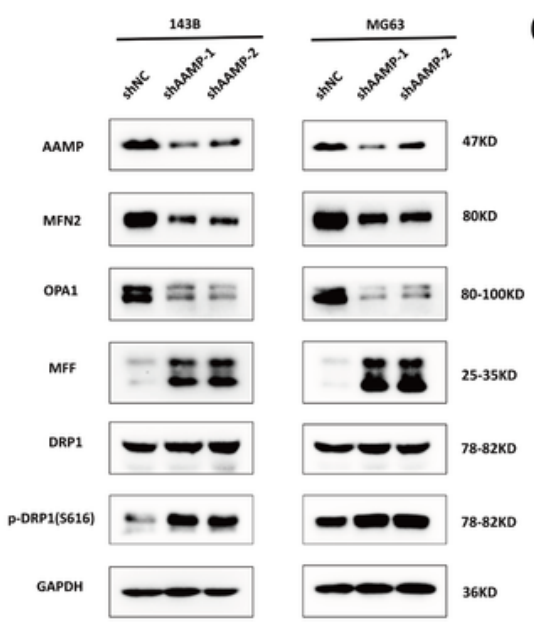

d
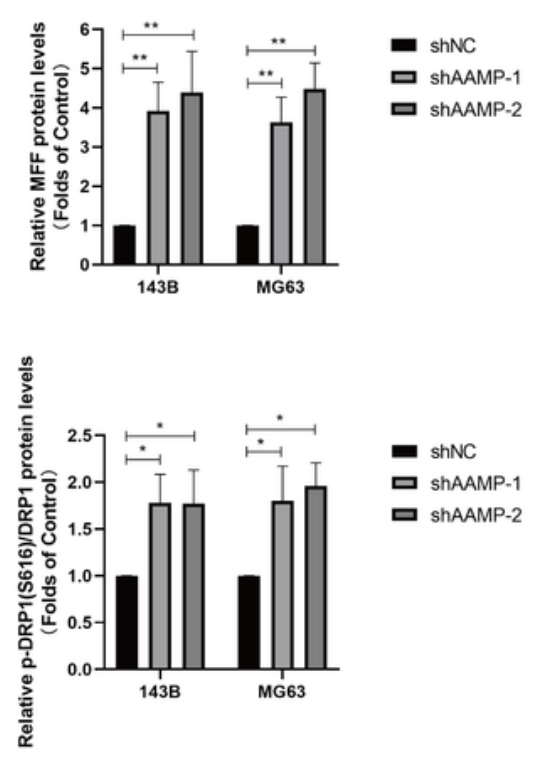

C
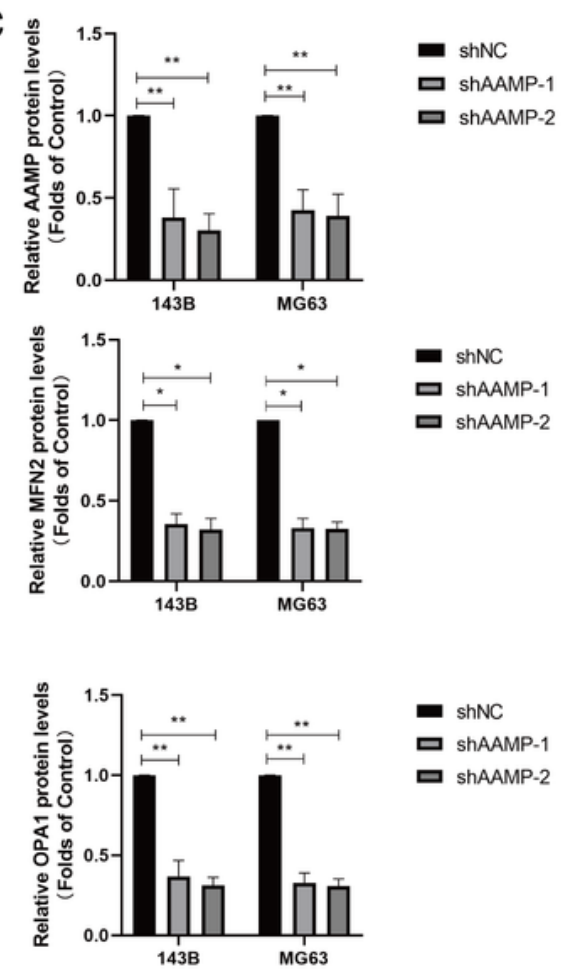

e

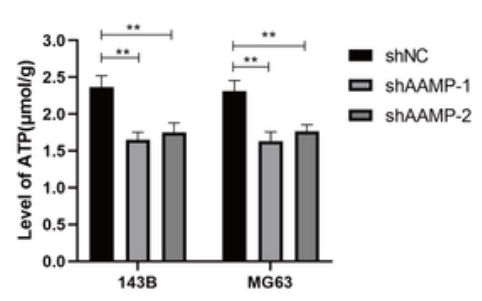

\section{Figure 4}

Knocking down AAMP induces mitochondrial dysfunction and decreases ATP production in OS cells. a Mitochondrial damage characterized by vacuolization and the loss or rupture of mitochondrial cristae (red arrow) was evident in cells in which AAMP had been knocked down but not in control cells in which mitochondria exhibited a clear double membrane and intact cristae (green arrow). b-d Mitochondriaassociated proteins were assessed via Western blotting following AAMP knockdown, with GAPDH as a 
loading control. e ATP levels were measured using an ATP Assay Kit. All analyses were repeated in triplicate. ${ }^{*} \mathrm{P}<0.05 * * \mathrm{P}<0.01 * * * \mathrm{P}<0.001$
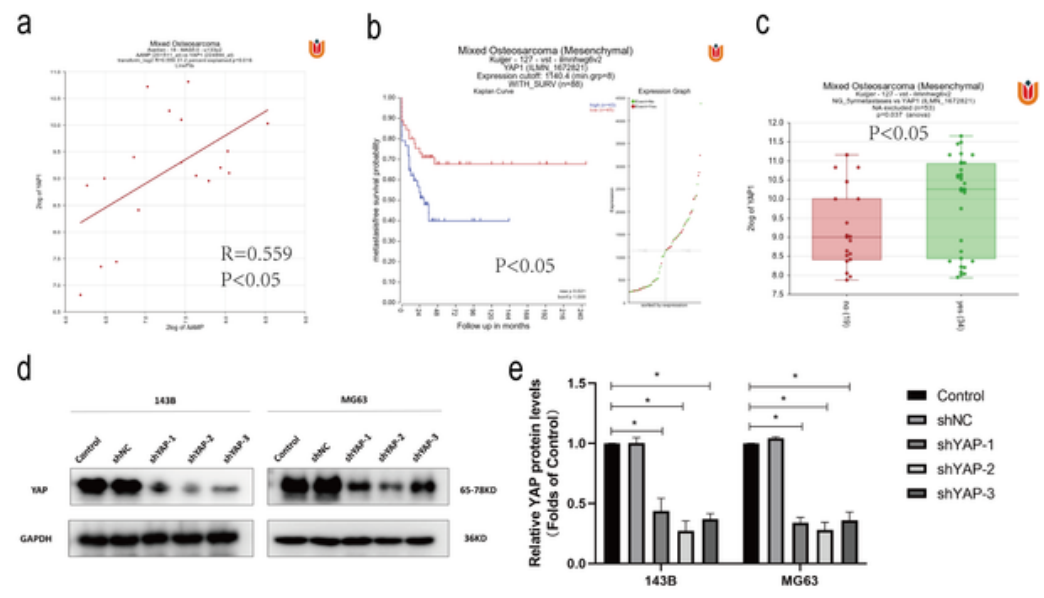

f

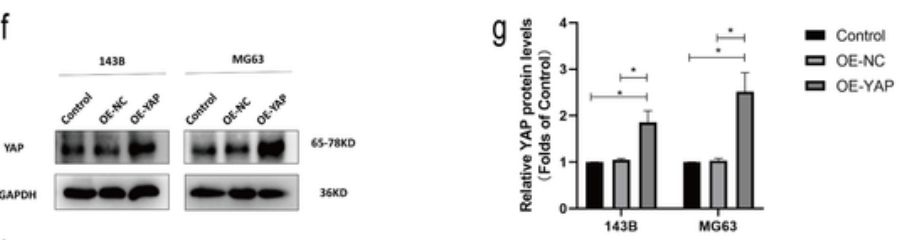

h
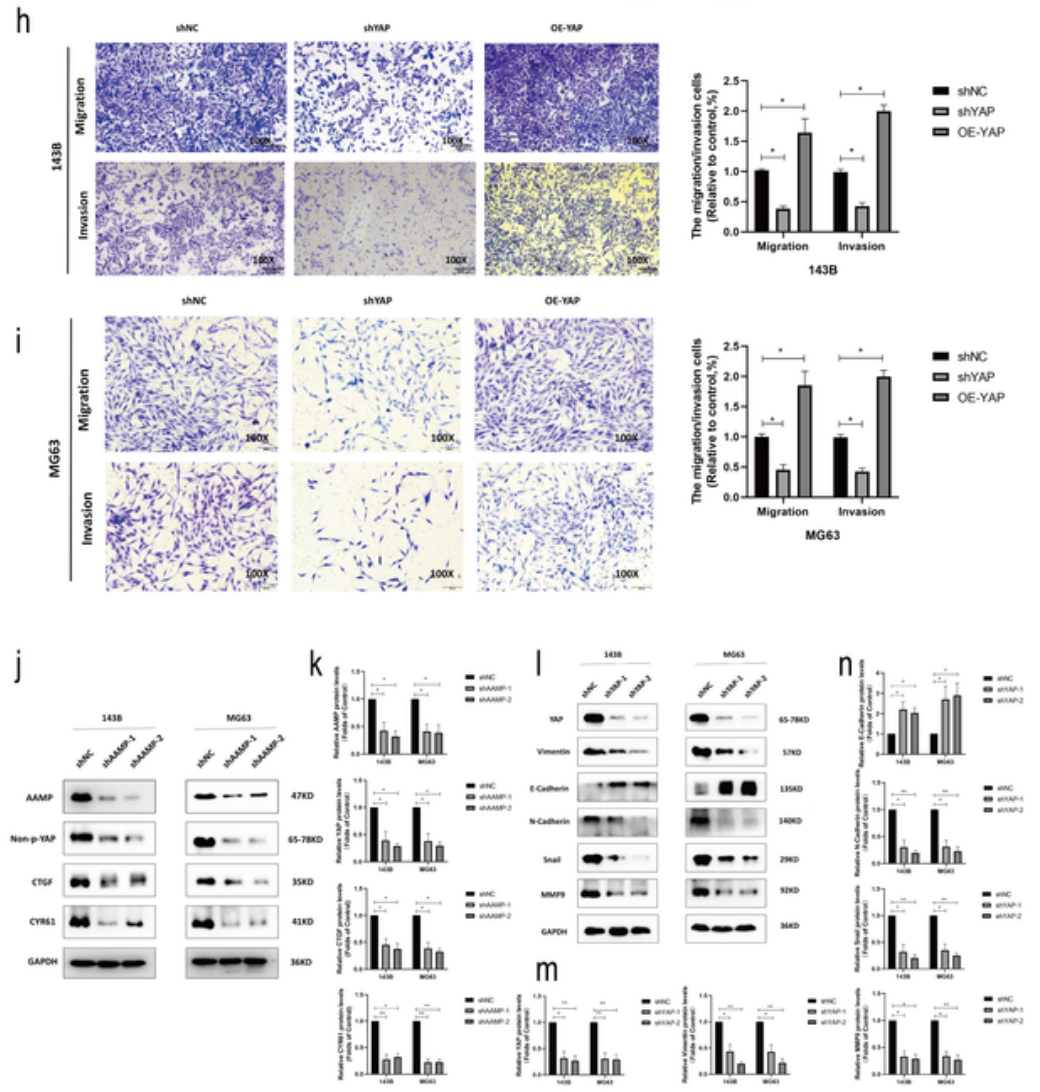

Figure 5

Knocking down AAMP suppresses OS cell migratory and invasive activity as a consequence of YAP inhibition. a A strong positive correlation between AAMP and YAP expression levels was observed in OS 
samples $(r=0.559, P<0.05)$. b Metastasis-free survival rates were assessed for 43 and 45 patients respectively exhibiting high and low YAP expression levels. c The gene expression level of YAP in metastatic osteosarcoma was compared with patients without metastasis. $\mathbf{d}-\mathbf{g}$ YAP levels were assessed in the indicated groups of 143B and MG63 levels via Western blotting, with GAPDH serving as a loading control. $\mathbf{h}, \mathbf{i}$ Transwell assays were conducted using 143B and MG63 cells following the knockdown or overexpression of AAMP. $\mathbf{j}$, $\mathbf{k}$ Western blotting was used to measure levels of YAP and associated downstream targets following the knockdown of YAP, with GAPDH serving as a loading control. I-n Western blotting was used to assess levels of EMT marker proteins in OS cells in which YAP had been knocked down. All analyses were conducted in triplicate. ${ }^{*} P<0.05 * * P<0.01 * \star * P<0.001$

a
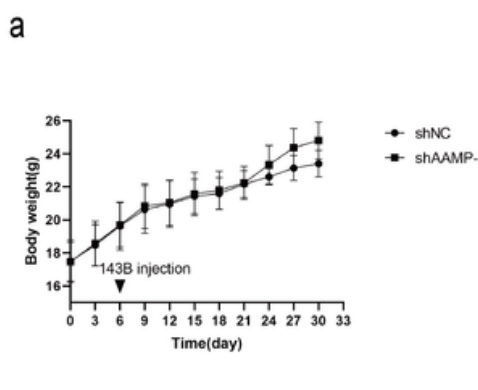

b
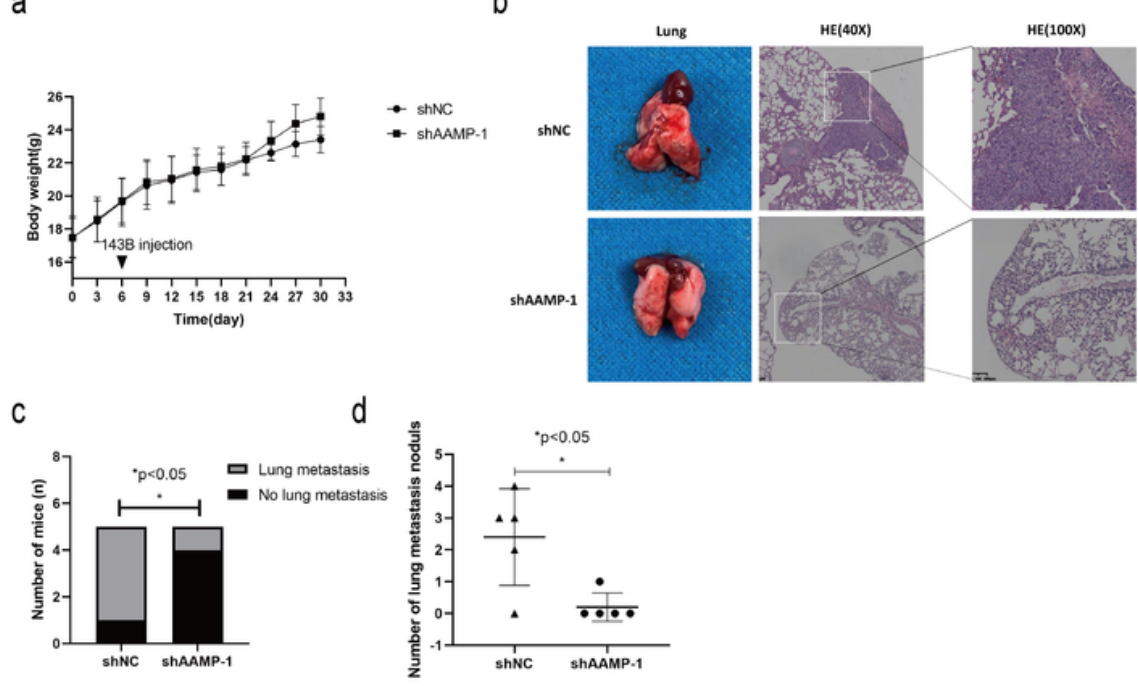

e

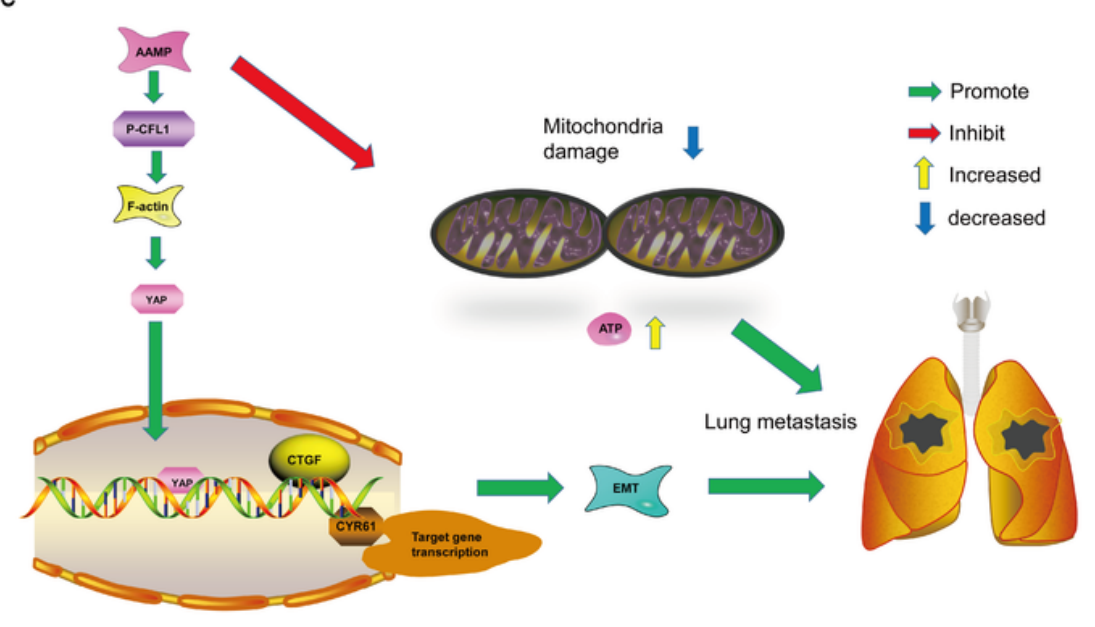

\section{Figure 6}

The knockdown of AAMP suppresses in vivo OS cell metastasis. a Nude mice were injected via the tail vein with 143B cells that had been engineered to stably express shNC or shAAMP ( $n=5 /$ group), with bodyweight values then being measured over time. $\mathbf{b}$ Macroscopic and microscopic images of pulmonary 
metastatic nodules that had been subjected to H\&E staining. c The percentages of mice in each group harboring lung metastases. $\mathbf{d}$ The numbers of metastatic lung nodules observed in H\&E-stained lung tissue sections. e The potential mechanisms whereby AAMP promotes OS cell metastasis to the lungs. AAMP can promote CFL1 phosphorylation and mitochondrial functionality, thereby driving YAP activation and enhancing ATP production, ultimately contributing to higher odds of lung metastasis.

\section{Supplementary Files}

This is a list of supplementary files associated with this preprint. Click to download.

- SupplementaryMaterialtables.docx 